\title{
Research Article Solving Nonlinear Partial Differential Equations by the sn-ns Method
}

\author{
Alvaro H. Salas \\ FIZMAKO Research Group and Department of Mathematics, University of Caldas/ National \\ University of Colombia, Campus la Nubia, Manizales, Colombia \\ Correspondence should be addressed to Alvaro H. Salas, asalash2002@yahoo.com \\ Received 2 January 2012; Accepted 30 January 2012 \\ Academic Editor: Muhammad Aslam Noor \\ Copyright (C) 2012 Alvaro H. Salas. This is an open access article distributed under the Creative \\ Commons Attribution License, which permits unrestricted use, distribution, and reproduction in \\ any medium, provided the original work is properly cited.
}

We present the application of the sn-ns method to solve nonlinear partial differential equations. We show that the well-known tanh-coth method is a particular case of the sn-ns method.

\section{Introduction}

The search of explicit solutions to nonlinear partial differential equations (NLPDEs) by using computational methods is one of the principal objectives in nonlinear science problems. Some powerful methods have been extensively used in the past decade to handle nonlinear PDEs. Some of them are the tanh-method [1], the tanh-coth method [2], the exp-function method [3], the projective Riccati equation method [4], and the Jacobi elliptic functions method. Practically, there is no unified method that could be used to handle all types of nonlinear problems.

The main purpose of this work consists in solving nonlinear polynomial PDE starting from the idea of the projective Riccati equations method. We derive exact solutions to the following equations: Duffing equation, cubic nonlinear Schrodinger equation, KleinGordon-Zakharov equations, quadratic Duffing equation, KdV equation, Gardner equation, Boussinesq equation, symmetric regular long wave equation, generalized shallow water wave equation, Klein-Gordon equation with quadratic nonlinearity, Fitzhugh-NagumoHuxley equation, and double sine-Gordon equation. 


\section{The Main Idea}

In the search of the traveling wave solutions to nonlinear partial differential equation of the form

$$
P\left(u, u_{x}, u_{t}, u_{x x}, u_{x t}, u_{t t}, \ldots\right)=0
$$

the first step consists in considering the wave transformation

$$
u(x, t)=v(\phi(\xi)), \quad \xi=x+\lambda t+\xi_{0}, \xi_{0}=\text { arbitrary constant, }
$$

for a suitable function $\phi=\phi(\xi)$, where $\lambda$ is a constant. Usually, $\phi(\xi)=\xi$ (the identity function).

Using (2.2), (2.1) converts to an ordinary differential equation (ODE) with respect to (shortly, w.r.t.) the function $v(\xi)$

$$
Q\left(v, v^{\prime}, v^{\prime \prime}, \ldots\right)=0
$$

with $Q$ being a polynomial with respect to variables $v, v^{\prime}, v^{\prime \prime}, \ldots$.

To find solutions to (2.3), we suppose that $v(\xi)$ can be expressed as

$$
v(\xi)=H(f(\xi), g(\xi))
$$

where $H(f, g)$ is a rational function in the new variables $f=f(\xi), g=g(\xi)$, which satisfy the system

$$
\begin{gathered}
f^{\prime}(\xi)=r f(\xi) g(\xi), \\
g^{2}(\xi)=S(f(\xi)),
\end{gathered}
$$

with $r \neq 0$ being some constant to be determined and $S(f)$ a rational function in the variable $f=f(\xi)$. We show that the system (2.5) may be solved exactly in certain cases. In fact, taking

$$
f(\xi)=\varphi^{N}(\xi),
$$

where $\varphi(\xi) \neq 0$ and $N \neq 0$, system (2.5) reduces to

$$
\begin{aligned}
& \varphi^{\prime}(\xi)=\frac{r}{N} \varphi(\xi) g(\xi), \\
& g^{2}(\xi)=S\left(\varphi^{N}(\xi)\right) .
\end{aligned}
$$

From (2.7) we get

$$
\left(\varphi^{\prime}(\xi)\right)^{2}=\frac{r^{2}}{N^{2}} \varphi^{2}(\xi) S\left(\varphi^{N}\right)
$$


Equation (2.8) is of elliptic type. Choosing $S(f)$ and $N$ adequately, we may obtain distinct methods to solve nonlinear PDEs. More exactly, suppose we have solved (2.8). Then, in view of (2.5) and (2.6) functions $f$ and $g$ may be computed by formulae

$$
f(\xi)=\varphi^{N}(\xi), \quad g(\xi)=\frac{f^{\prime}(\xi)}{r f^{\prime}(\xi)}=\frac{N}{r} \frac{\varphi^{\prime}(\xi)}{\varphi(\xi)} .
$$

To solve (2.3) we try one of the following ansatz:

$$
\begin{gathered}
v(\xi)=a_{0}+\sum_{j=1}^{n} f^{j-1}\left(a_{j} f+b_{j} g\right), \\
v(\xi)=a_{0}+\sum_{j=1}^{n} a_{j} f^{j}, \\
v(\xi)=a_{0}+\sum_{j=1}^{n}\left(a_{j} f^{j}+b_{j} f^{-j}\right) .
\end{gathered}
$$

We substitute any of these ansatz into (2.3) and we obtain a polynomial equation either in the variables $f=f(\xi)$ and $g=g(\xi)$ or $f=f(\xi)$. We equate the coefficients of $f^{i} g^{j}(i, j=0,1,2,3, \ldots)$ to zero, and we obtain a system of polynomial equations in the variables $a_{j}, b_{i}, \lambda, \ldots$ Solving this system with the aid of a symbolic computational package such as Mathematica 8 or Maple 15, we obtain the desired solutions. Sometimes, we replace $\xi$ with $k \xi$ and then the corresponding system is regarded w.r.t. the variables $f=f(k \xi)$ and $g=g(k \xi)$, where $k=$ const.

We also may solve coupled systems of nonlinear equations. Indeed, suppose that we have a coupled system of two equations in the form

$$
\begin{aligned}
& P\left(u, \tilde{u}, u_{x}, \tilde{u}_{x}, u_{t}, \tilde{u}_{t}, u_{x x}, \tilde{u}_{x x}, u_{x t}, \tilde{u}_{x t}, u_{t t}, \tilde{u}_{t t}, \ldots\right)=0, \\
& \widetilde{P}\left(u, \tilde{u}_{1} u_{x}, \tilde{u}_{x}, u_{t}, \tilde{u}_{t}, u_{x x}, \tilde{u}_{x x}, u_{x t}, \tilde{u}_{x t}, u_{t t}, \tilde{u}_{t t}, \ldots\right)=0 .
\end{aligned}
$$

We first apply the wave transformation

$$
\begin{aligned}
& u(x, t)=v(\phi(\xi)), \quad \tilde{u}(x, t)=\tilde{v}(\tilde{\phi}(\xi)), \\
& \xi=x+\lambda t+\xi_{0}, \quad \xi_{0}=\text { arbitrary constant, }
\end{aligned}
$$

for a suitable pair of functions $\phi=\phi(\xi)$ and $\tilde{\phi}=\tilde{\phi}(\xi)$ in order to obtain a coupled system of two ODEs in the form

$$
\begin{aligned}
& Q\left(v, \widetilde{v}, v^{\prime}, \tilde{v}^{\prime}, v^{\prime \prime}, \tilde{v}^{\prime \prime}, v^{\prime \prime \prime}, \tilde{v}^{\prime \prime \prime}, \ldots\right)=0, \\
& \tilde{Q}\left(v, \widetilde{v}, v^{\prime}, \tilde{v}^{\prime}, v^{\prime \prime}, \widetilde{v}^{\prime \prime}, v^{\prime \prime \prime}, \widetilde{v}^{\prime \prime \prime}, \ldots\right)=0,
\end{aligned}
$$

where $Q$ and $\widetilde{Q}$ are polynomials w.r.t. the variables $v, \widetilde{v}, v^{\prime}, \widetilde{v}^{\prime}, v^{\prime \prime}, \widetilde{v}^{\prime \prime}, v^{\prime \prime \prime}, \widetilde{v}^{\prime \prime \prime}, \ldots$ 
We seek solutions to system (2.14) in the forms

$$
\begin{gathered}
v(\xi)=a_{0}+\sum_{j=1}^{n} f^{j-1}\left(a_{j} f+b_{j} g\right), \\
\tilde{v}(\xi)=\tilde{a}_{0}+\sum_{j=1}^{\tilde{n}} f^{j-1}\left(\tilde{a}_{j} f+\tilde{b}_{j} g\right), \\
v(\xi)=a_{0}+\sum_{j=1}^{n} a_{j} f^{j}, \\
\tilde{v}(\xi)=\tilde{a}_{0}+\sum_{j=1}^{\tilde{n}} \tilde{a}_{i} f^{i}, \\
v(\xi)=a_{0}+\sum_{j=1}^{n}\left(a_{j} f^{j}+b_{j} f^{-j}\right), \\
\tilde{v}(\xi)=\tilde{a}_{0}+\sum_{i=1}^{\tilde{n}}\left(\tilde{a}_{i} f^{i}+\tilde{b}_{i} f^{-i}\right) .
\end{gathered}
$$

The integers $n$ and $\tilde{n}$ are determined by the balancing method.

We substitute any of ansatz (2.15), (2.16), or (2.17) into (2.14), and we obtain a system of two polynomial equations either w.r.t. the variables $f=f(\xi)$ and $g=g(\xi)$ or w.r.t. the variable $f=f(\xi)$. We equate the coefficients of $f^{i} g^{j}$ (resp., the coefficients of $f^{i}$ ) $(i, j=0,1,2,3, \ldots)$ to zero, and we obtain a system of polynomial equations w.r.t the variables $a_{i}, b_{j}, \tilde{a}_{i}, \widetilde{b}_{j}, \lambda, \ldots$ Solving this system with the aid of a symbolic computational package such as Mathematica or Maple, we obtain the desired solutions. Sometimes, with the aim to add an additional parameter $k$, we replace $\xi$ with $k \xi$ and then the corresponding system is regarded w.r.t. the variables $f=f(k \xi)$ and $g=g(k \xi)$, where $k=$ const.

The same technique is applied for solving systems of three or more equations.

\section{The sn-ns Method and Its Derivation}

Let $N=-1, r=1$ and $S(f)=a f^{-2}+b+c f^{2}$, where

$$
\Delta=b^{2}-4 a c>0
$$

This choice gives us (2.8) in the form

$$
\left(\varphi^{\prime}\right)^{2}=a \varphi^{4}+b \varphi^{2}+c
$$


We may express the general solution of this equation in terms of the Jacobi elliptic functions ns or nd as follows:

$$
\varphi(\xi)= \pm \sqrt{\frac{-b+\sqrt{\Delta}}{2 a}} \mathrm{~ns}(k(\xi+C) \mid m), \quad C=\text { arbitrary constant }
$$

where

$$
k=\sqrt{\frac{-b+\sqrt{\Delta}}{2}}, \quad m=\sqrt{\frac{b^{2}-2 a c+b \sqrt{\Delta}}{2 a c}} .
$$

Solution (3.3) is valid for $a>0, b<0$, and $0<c \leq b^{2} / 4 a$.

On the other hand, function $\xi \rightarrow \sqrt{-1} \mathrm{~ns}(\sqrt{-1} k(\xi+C) \mid m)$ is real valued for any real $k, m, \xi$, and $C$. We may verify that function

$$
\phi(\xi)= \pm \sqrt{-1} \sqrt{\frac{-b+\sqrt{\Delta}}{2 a}} \mathrm{~ns}\left(\sqrt{-1} k\left(\xi+\xi_{0}\right) \mid m\right),
$$

where $k$ and $m$ are given by (3.4), is a solution to equation

$$
\left(\varphi^{\prime}\right)^{2}=a \varphi^{4}-b \varphi^{2}+c, \quad a>0, b<0,0<c \leq \frac{b^{2}}{4 a} .
$$

Thus, we always may find a solution to (3.2) when $a>0$ and $0<c \leq b^{2} / 4 a$ for any $b \neq 0$.

Now, let us assume that $a<0$. It may be verified that a solution to (3.2) is

$$
\varphi(\xi)= \pm \sqrt{\frac{-b-\sqrt{\Delta}}{2 a}} \operatorname{nd}(k(\xi+C) \mid m), \quad C=\text { arbitrary constant }
$$

where

$$
k=\sqrt{\frac{b+\sqrt{\Delta}}{2}}, \quad m=\sqrt{-\frac{b^{2}-4 a c+b \sqrt{\Delta}}{2 a c}}, \quad \text { for } b \neq 0, c>0 .
$$

Observe that for any $A>0$ function $\xi \rightarrow \mathrm{nd}(\sqrt{-A}(\xi+C) \mid m)$ is real valued for any real values of $m, \xi$, and $C$. We conclude that (3.2) has exact solutions for any $a \neq 0$ and $b \neq 0$.

Projective equations are

$$
\begin{gathered}
f^{\prime}(\xi)=f(\xi) g(\xi), \\
g^{2}(\xi)=\frac{a}{f^{2}(\xi)}+b+c f^{2}(\xi) .
\end{gathered}
$$


Taking $C=0$ in (3.3) we see that solutions to system (3.9) are

$$
f(\xi)=\varphi^{-1}(\xi)=\frac{\sqrt{a}}{k} \operatorname{sn}(k \xi \mid m), \quad g(\xi)=\frac{f^{\prime}(\xi)}{f(\xi)}=k \operatorname{sn}(k \xi \mid m) \operatorname{cs}(k \xi \mid m) \operatorname{ds}(k \xi \mid m) .
$$

This motivates us to seek solutions to (2.3) in the form

$$
v(\xi)=a_{0}+\sum_{j=1}^{n}\left[a_{j} \mathrm{sn}^{j}(k \xi)+b_{j} \mathrm{~ns}^{j}(k \xi)\right]
$$

Usually, $n=1,2$ and then $v(\xi)$ has the forms

$$
\begin{gathered}
v(\xi)=a_{0}+a_{1} \operatorname{sn}(k \xi \mid m)+b_{1} \operatorname{ns}(k \xi \mid m), \\
v(\xi)=a_{0}+a_{1} \operatorname{sn}(k \xi \mid m)+b_{1} \operatorname{ns}(k \xi \mid m)+a_{2} \operatorname{sn}^{2}(k \xi \mid m)+b_{2} \mathrm{~ns}^{2}(k \xi \mid m) .
\end{gathered}
$$

In the case when $m=1$, this gives the tanh-coth method since $\operatorname{sn}(k \xi \mid 1)=\tanh (k \xi)$ and $\mathrm{ns}(k \xi \mid 1)=\operatorname{coth}(k \xi)$.

Another possible ansatz suggested from (2.10) is

$$
v(\xi)=a_{0}+\sum_{j=1}^{n} \operatorname{sn}^{j-1}(k \xi)\left[a_{j} \operatorname{sn}(k \xi \mid m)+b_{j} \operatorname{sn}(k \xi \mid m) \operatorname{cs}(k \xi \mid m) \operatorname{ds}(k \xi \mid m)\right]
$$

If $n=1,2$, this ansatz reads

$$
\begin{aligned}
v(\xi)= & a_{0}+a_{1} \operatorname{sn}(k \xi \mid m)+b_{1} \operatorname{sn}(k \xi \mid m)+\operatorname{cs}(k \xi \mid m)+\operatorname{ds}(k \xi \mid m) \\
v(\xi)= & a_{0}+a_{1} \operatorname{sn}(k \xi \mid m)+b_{1} \operatorname{sn}(k \xi \mid m) \operatorname{cs}(k \xi \mid m) \operatorname{ds}(k \xi \mid m) \\
& +a_{2} \operatorname{sn}^{2}(k \xi \mid m)+b_{2} \operatorname{sn}^{2}(k \xi \mid m) \operatorname{cs}(k \xi \mid m) \operatorname{ds}(k \xi \mid m) .
\end{aligned}
$$

We may consider similar ansatz by replacing sn by dn and ns by nd, respectively. We will call this the dn-nd method. Thus, two possible ansatz for this method are

$$
\begin{gathered}
v(\xi)=a_{0}+a_{1} \operatorname{dn}(k \xi \mid m)+b_{1} \operatorname{nd}(k \xi \mid m), \\
v(\xi)=a_{0}+a_{1} \operatorname{dn}(k \xi \mid m)+b_{1} \operatorname{nd}(k \xi \mid m)+a_{2} \operatorname{dn}^{2}(k \xi \mid m)+b_{2} \operatorname{nd}^{2}(k \xi \mid m) .
\end{gathered}
$$

An other useful ansatz to handle other equations of the form (2.3) is

$$
v(\xi)=a_{0}+a_{1} \mathrm{cn}(k \xi \mid m)+b_{1} \mathrm{nc}(k \xi \mid m) .
$$


We will call this the cn-nc method.

We also may try the following ansatz:

$$
v(\xi)=\frac{a_{0}+a_{1} \mathrm{cn}(k \xi \mid m)}{1+b_{1} \mathrm{cn}(k \xi \mid m)}
$$

For example, this ansatz may be successfully applied to the cubic-quintic Duffing equation, which is defined by

$$
v^{\prime \prime}(\xi)+p v(\xi)+q v^{3}(\xi)+r v^{5}(\xi)=0
$$

\section{Examples}

In this section we solve various important models related to nonlinear science by the methods described in previous sections.

\subsection{Duffing Equation $v^{\prime \prime}(\xi)+p v(\xi)+q v^{3}(\xi)=0$}

Let us consider the equation

$$
v^{\prime \prime}(\xi)+p v(\xi)+q v^{3}(\xi)=0
$$

where $p$ and $q$ are nonzero constants. This equation is very important since some relevant physical models described by a nonlinear PDEs may be studied once this equation is solved. Two of them are related to cubic nonlinear the Schrodinger equation and the Klein-GordonZakharov equations. equation is

To find solutions we multiply (4.1) by $v^{\prime}(\xi)$ and we integrate it w.r.t. $\xi$. The resulting

$$
\left(\frac{d v}{d \xi}\right)^{2}=\frac{q}{2} v^{4}(\xi)-p v^{2}(\xi)-2 C
$$

where $C$ is the constant of integration. This equation has form (3.2) and we already know that there exists an exact solution to it for any $p$ and $q$. Instead, we may apply directly the sn-ns method (resp., the dn-nd method) to it. Balancing gives $n=1$. We seek solutions in the form

$$
v(\xi)=a_{0}+a_{1} \operatorname{sn}(k \xi \mid m)+b_{1} \operatorname{ns}(k \xi \mid m) .
$$


Inserting (4.3) into (4.1), we obtain a polynomial equation w.r.t. the variable $\zeta=\operatorname{sn}(k \xi \mid m)$. Equating to zero the coefficients of $\zeta^{j}(j=0,1,2, \ldots)$ yields the following algebraic system:

$$
\begin{gathered}
3 a_{0} a_{1}^{2} q=0, \\
a_{1}\left(a_{1}^{2} q+2 k^{2} m^{2}\right)=0, \\
a_{1} b_{1}\left(3 a_{1} b_{1} q+3 a_{0}^{2} q-k^{2} m^{2}-k^{2}+p\right)=0, \\
a_{0}\left(6 a_{1} b_{1} q+a_{0}^{2} q+p\right)=0, \\
b_{1}\left(b_{1}^{2} q+2 k^{2}\right)=0 .
\end{gathered}
$$

Solving system (4.4) gives solutions as follows:

$$
\begin{aligned}
& \text { (i) } a_{0}=0, a_{1}=\sqrt{-2 p} m / \sqrt{\left(m^{2}+6 m+1\right) q}, b_{1}=\sqrt{-2 p} / \sqrt{\left(m^{2}+6 m+1\right) q}, k= \\
& \sqrt{p} / \sqrt{m^{2}+6 m+1} \\
& v(\xi)= \pm \frac{\sqrt{-2 p}}{\sqrt{\left(m^{2}+6 m+1\right) q}}\left(m \operatorname{sn}\left(\frac{\sqrt{p}}{\sqrt{m^{2}+6 m+1}} \xi \mid m\right)+\mathrm{ns}\left(\frac{\sqrt{p}}{\sqrt{m^{2}+6 m+1}} \xi \mid m\right)\right) \\
& v(\xi)= \pm \frac{\sqrt{-2 p}}{\sqrt{\left(m^{2}-6 m+1\right) q}}\left(m \operatorname{sn}\left(\frac{\sqrt{p}}{m^{2}-6 m+1} \xi \mid m\right)-\mathrm{ns}\left(\frac{\sqrt{p}}{\sqrt{m^{2}-6 m+1}} \xi \mid m\right)\right) \\
& v(\xi)= \pm m \sqrt{-\frac{2 p}{q\left(m^{2}+1\right)}} \operatorname{sn}\left(\sqrt{\frac{p}{m^{2}+1}} \xi \mid m\right)
\end{aligned}
$$

(iii) $a_{0}=0, a_{1}=0, b_{1}=\sqrt{-2 p} / \sqrt{q\left(m^{2}+1\right)}, k=\sqrt{p} / \sqrt{m^{2}+1}$,

$$
v(\xi)= \pm \frac{\sqrt{-2 p}}{\sqrt{q\left(m^{2}+1\right)}} \mathrm{ns}\left(\frac{\sqrt{p}}{\sqrt{m^{2}+1}} \xi \mid m\right)
$$


Letting $m \rightarrow 1$, we obtain trigonometric and hyperbolic solutions:

$$
\begin{gathered}
v(\xi)=\sqrt{-\frac{p}{2 q}} \tanh \left(\frac{\sqrt{p}}{2 \sqrt{2}} \xi\right)+\sqrt{-\frac{p}{2 q}} \operatorname{coth}\left(\frac{\sqrt{p}}{2 \sqrt{2}} \xi\right), \\
v(\xi)=-\sqrt{-\frac{p}{2 q}} \tan \left(\frac{\sqrt{p}}{2} \xi\right)-\sqrt{-\frac{p}{2 q}} \cot \left(\frac{\sqrt{p}}{2} \xi\right)=\sqrt{-\frac{2 p}{q}} \csc (\sqrt{p \xi}), \\
v(\xi)= \pm \sqrt{-\frac{p}{q}} \operatorname{coth}\left(\sqrt{\frac{p}{2}} \xi\right), \\
v(\xi)= \pm \sqrt{-\frac{p}{q}} \tanh \left(\sqrt{\frac{p}{2}} \xi\right) .
\end{gathered}
$$

\subsection{Cubic Nonlinear Schrodinger Equation}

This equation reads

$$
i u_{t}+u_{x x}+\mu|u|^{2} u=0,
$$

where $u=u(x, t)$ is a complex-valued function of two real variables $x$ and $t$ and $\mu$ is a nonzero real parameter and $i=\sqrt{-1}$. The physical model of the cubic nonlinear Schrodinger equation (shortly, NLS equation) (4.11) and its generalized variants occur in various areas of physics such as nonlinear optics, water waves, plasma physics, quantum mechanics, superconductivity, and the Bose-Einstein condensate theory. It also has applications in optics since it models many nonlinearity effects in a fiber, including but not limited to self-phase modulation, four-wave mixing, second harmonic generation, stimulated Raman scattering, and so forth. For water waves, the NLS equation (4.11) describes the evolution of the envelope of modulated nonlinear wave groups. All these physical phenomena can be better understood with the help of exact solutions for a given $\mu$. When $\mu>0$, the equation is said to be attractive. If $\mu<0$ we say that it is repulsive. Recently, Ma and Chen [5] obtained some solutions to (4.11).

We seek solutions to (4.11) in the form

$$
u(x, t)=v(\xi) \exp (i(\alpha x+\gamma t)), \quad \xi=x-2 \alpha t+\xi_{0},
$$

where $\alpha$ and $\gamma$ are some real constants to be determined. Inserting (4.12) into (4.11) and simplifying, we obtain

$$
v^{\prime \prime}(\xi)-\left(\gamma+\alpha^{2}\right) v(\xi)+\mu v^{3}(\xi)=0
$$


This last equation has the form (4.1) with

$$
p=-\left(\gamma+\alpha^{2}\right), \quad q=\mu .
$$

Thus, making use of solutions (4.5)-(4.10) for the choices given by (4.14) we obtain exact solutions to the Schrodinger equation (4.11) in the form (4.12).

\subsection{Quadratic Duffing Equation $v^{\prime \prime}(\xi)=p v^{2}(\xi)+q v(\xi)+r$}

Let us consider the following second-order and second-degree nonlinear ODE:

$$
v^{\prime \prime}(\xi)=p v^{2}(\xi)+q v(\xi)+r
$$

where $p, q$, and $r$ are constants and $p \neq 0$. Solutions to this equation may be used to study some important physical models whose associated PDEs may be solved after making the traveling wave transformation (2.2). As we will show in next subsections some examples of nonlinear partial differential equations where this equation arises are the following.

(i) KdV equation: $u_{t}+6 u u_{x}+u_{x x x}=0$.

(ii) Gardner equation (also called combined KdV-mKdV equation): $u_{t}+\alpha u u_{x}+\beta u^{2} u_{x}+$ $\gamma u_{x x x}=0, \beta \gamma \neq 0$,

(iii) Boussinesq equation: $u_{t t}+\alpha u u_{x x}+\alpha u_{x}^{2}+\beta u_{x x x x}=0$,

(iv) symmetric regular long wave equation: $u_{t t}+u_{x x}+u u_{x t}+u_{x} u_{t}+u_{x x t t}=0$,

(v) generalized shallow water wave equation: $u_{x x t t}+\alpha u_{x} u_{x t}+\beta u_{t} u_{x x}-u_{x t}-u_{x x}=0$,

(vi) Klein-Gordon equation with quadratic nonlinearity: $u_{t t}-\alpha^{2} u_{x x}+\beta u-\gamma u^{2}=0$.

Balancing gives $n=2$. We seek solutions to (4.15) in the form (3.13), that is,

$$
v(\xi)=a_{0}+a_{1} \operatorname{sn}(k \xi \mid m)+b_{1} \mathrm{~ns}(k \xi \mid m)+a_{2} \operatorname{sn}^{2}(k \xi \mid m)+b_{2} \mathrm{~ns}^{2}(k \xi \mid m) .
$$


Inserting this ansatz into (4.15) gives the following algebraic system:

$$
\begin{gathered}
a_{1}\left(k^{2} m^{2}-a_{2} p\right)=0 \\
a_{2}\left(6 k^{2} m^{2}-a_{2} p\right)=0 \\
4 a_{2} k^{2} m^{2}+4 a_{2} k^{2}+a_{1}^{2} p+2 a_{0} a_{2} p+a_{2} q=0 \\
2 a_{2} b_{1} p+a_{1} k^{2} m^{2}+a_{1} k^{2}+2 a_{0} a_{1} p+a_{1} q=0 \\
b_{1}\left(k^{2}-b_{2} p\right)=0 \\
b_{2}\left(6 k^{2}-b_{2} p\right)=0 \\
2 a_{0} b_{2} p+4 b_{2} k^{2} m^{2}+4 b_{2} k^{2}+b_{1}^{2} p+b_{2} q=0 \\
2 a_{0} b_{1} p+2 a_{1} b_{2} p+b_{1} k^{2} m^{2}+b_{1} k^{2}+b_{1} q=0 \\
2 a_{1} b_{1} p+2 a_{2} b_{2} p-2 a_{2} k^{2}+a_{0}^{2} p+a_{0} q-2 b_{2} k^{2} m^{2}+r=0
\end{gathered}
$$

Solving this system, we obtain the following solutions:

(i) $k=(1 / 2) \sqrt[4]{\left(q^{2}-4 p r\right) /\left(m^{4}-m^{2}+1\right)}, \quad a_{0}=q / 2 p-(1 / 2 p)\left(m^{2}+\right.$ 1) $\sqrt{\left(q^{2}-4 p r\right) /\left(m^{4}-m^{2}+1\right)}, a_{1}=0, a_{2}=\left(3 m^{2} / 2 p\right) \sqrt{\left(q^{2}-4 p r\right) /\left(m^{4}-m^{2}+1\right)}, b_{1}=$ $0, b_{2}=0$,

$$
v(\xi)=a_{0}+\frac{3 m^{2}}{2 p} \sqrt{\frac{q^{2}-4 p r}{m^{4}-m^{2}+1}} \mathrm{sn}^{2}\left(\frac{1}{2} \sqrt[4]{\frac{q^{2}-4 p r}{m^{4}-m^{2}+1}} \xi \mid m\right)
$$

(ii) $k=(1 / 2) \sqrt[4]{\left(q^{2}-4 p r\right) /\left(m^{4}-m^{2}+1\right)}, \quad a_{0}=-q / 2 p-(1 / 2 p)\left(m^{2}+\right.$ 1) $\sqrt{\left(q^{2}-4 p r\right) /\left(m^{4}-m^{2}+1\right)}, \quad a_{1}=0, \quad a_{2}=0, \quad b_{1}=0, \quad b_{2}=$ $(3 / 2 p) \sqrt{\left(q^{2}-4 p r\right) /\left(m^{4}-m^{2}+1\right)}$,

$$
v(\xi)=a_{0}+\frac{3}{2 p} \sqrt{\frac{q^{2}-4 p r}{m^{4}-m^{2}+1}} \mathrm{~ns}^{2}\left(\frac{1}{2} \sqrt[4]{\frac{q^{2}-4 p r}{m^{4}-m^{2}+1}} \xi \mid m\right),
$$


(iii) $k=(1 / 2) \sqrt[4]{\left(q^{2}-4 p r\right) /\left(m^{4}+14 m^{2}+1\right)}, \quad a_{0}=-q / 2 p-(1 / 2 p)\left(m^{2}+\right.$ 1) $\sqrt{\left(q^{2}-4 p r\right) /\left(m^{4}-m^{2}+1\right)}, a_{1}=0, a_{2}=\left(3 m^{2} / 2 p\right) \sqrt{\left(q^{2}-4 p r\right) /\left(m^{4}+14 m^{2}+1\right)}$, $b_{1}=0, b_{2}=(3 / 2 p) \sqrt{\left(q^{2}-4 p r\right) /\left(m^{4}+14 m^{2}+1\right)}$,

$$
\begin{array}{r}
v(\xi)=a_{0}+\frac{3}{2 p} \sqrt{\frac{q^{2}-4 p r}{m^{4}+14 m^{2}+1}}\left(3 m^{2} \operatorname{sn}^{2}\left(\frac{1}{2} \sqrt[4]{\frac{q^{2}-4 p r}{m^{4}+14 m^{2}+1}} \xi \mid m\right)\right. \\
\left.+\mathrm{ns}^{2}\left(\frac{1}{2} \sqrt[4]{\frac{q^{2}-4 p r}{m^{4}+14 m^{2}+1}} \xi \mid m\right)\right),
\end{array}
$$

(iv) $k=(\sqrt{-1} / 2) \sqrt[4]{\left(q^{2}-4 p r\right) /\left(m^{4}-m^{2}+1\right)}, \quad a_{0} \quad=\quad(1 / 2 p)\left[\left(m^{2}+\right.\right.$ 1) $\left.\sqrt{\left(q^{2}-4 p r\right) /\left(m^{4}-m^{2}+1\right)}-q\right], \quad a_{1}=0, a_{2}=0, b_{1}=0, b_{2}=$ $(-3 / 2 p) \sqrt{\left(q^{2}-4 p r\right) /\left(m^{4}-m^{2}+1\right)}$,

$$
v(\xi)=a_{0}-\frac{3}{2 p} \sqrt{\frac{q^{2}-4 p r}{m^{4}-m^{2}+1}} \mathrm{~ns}^{2}\left(\frac{\sqrt{-1}}{2} \sqrt[4]{\frac{q^{2}-4 p r}{m^{4}-m^{2}+1}} \xi \mid m\right)
$$

(v) $k=(\sqrt{-1} / 2) \sqrt[4]{\left(q^{2}-4 p r\right) /\left(m^{4}-m^{2}+1\right)}, \quad a_{0}=q / 2 p-(1 / 2 p)\left(m^{2}+\right.$ 1) $\sqrt{\left(q^{2}-4 p r\right) /\left(m^{4}-m^{2}+1\right)}, a_{1}=0, a_{2}=-\left(3 m^{2} / 2 p\right) \sqrt{\left(q^{2}-4 p r\right) /\left(m^{4}-m^{2}+1\right)}, b_{1}=$ $0, b_{2}=0$,

$$
v(\xi)=a_{0}-\frac{3 m^{2}}{2 p} \sqrt{\frac{q^{2}-4 p r}{m^{4}-m^{2}+1}} \operatorname{sn}^{2}\left(\frac{\sqrt{-1}}{2} \sqrt[4]{\frac{q^{2}-4 p r}{m^{4}-m^{2}+1}} \xi \mid m\right),
$$

(vi) $k=(\sqrt{-1} / 2) \sqrt[4]{\left(q^{2}-4 p r\right) /\left(m^{4}+14 m^{2}+1\right)}, a_{0}=q / 2 p-(1 / 2 p)\left(m^{2}+\right.$

1) $\sqrt{\left(q^{2}-4 p r\right) /\left(m^{4}+14 m^{2}+1\right)}, a_{1}=0, a_{2}=-\left(3 m^{2} / 2 p\right) \sqrt{\left(q^{2}-4 p r\right) /\left(m^{4}+14 m^{2}+1\right)}$, $b_{1}=0, b_{2}=-(3 / 2 p) \sqrt{\left(q^{2}-4 p r\right) /\left(m^{4}+14 m^{2}+1\right)}$,

$$
\begin{array}{r}
v(\xi)=a_{0}-\frac{3}{2 p} \sqrt{\frac{q^{2}-4 p r}{m^{4}+14 m^{2}+1}}\left(3 m^{2} \operatorname{sn}^{2}\left(\frac{\sqrt{-1}}{2} \sqrt[4]{\frac{q^{2}-4 p r}{m^{4}+14 m^{2}+1}} \xi \mid m\right)\right. \\
\left.+\mathrm{ns}^{2}\left(\frac{\sqrt{-1}}{2} \sqrt[4]{\frac{q^{2}-4 p r}{m^{4}+14 m^{2}+1}} \xi \mid m\right)\right),
\end{array}
$$

Letting $m \rightarrow 1$, we obtain trigonometric and hyperbolic solutions: 
Abstract and Applied Analysis

$$
\begin{gathered}
v(\xi)=a_{0}+\frac{3}{2 p} \sqrt{q^{2}-4 p r} \tanh ^{2}\left(\frac{1}{2} \sqrt[4]{q^{2}-4 p r} \xi\right) . \\
v(\xi)=a_{0}+\frac{3}{2 p} \sqrt{q^{2}-4 p r} \operatorname{coth}^{2}\left(\frac{1}{2} \sqrt[4]{q^{2}-4 p r} \xi\right) . \\
v(\xi)=a_{0}+\frac{3}{4 p} \sqrt{q^{2}-4 p r}\left(3 \tanh ^{2}\left(\frac{1}{4} \sqrt[4]{q^{2}-4 p r} \xi\right)+\operatorname{coth}^{2}\left(\frac{1}{4} \sqrt[4]{q^{2}-4 p r} \xi\right)\right) .
\end{gathered}
$$

Now, let us seek solutions in the ansatz form (3.16), that is,

$$
\begin{aligned}
v(\xi)= & a_{0}+a_{1} \operatorname{sn}(k \xi \mid m)+b_{1} \operatorname{ns}(k \xi \mid m) \operatorname{cn}(k \xi \mid m) \operatorname{dn}(k \xi \mid m) \\
& +a_{2} \operatorname{sn}^{2}(k \xi \mid m)+b_{2} \operatorname{cn}(k \xi \mid m) \operatorname{dn}(k \xi \mid m) .
\end{aligned}
$$

Inserting this ansatz into (4.15) and solving the resulting algebraic system yields the following solutions to (4.15):

$$
\begin{aligned}
& \text { (i) } k=\sqrt{-1} \sqrt[4]{\left(q^{2}-4 p r\right) /\left(m^{4}+14 m^{2}+1\right)}, \quad a_{0}=-q / 2 p+(1 / 2 p)\left(m^{2}+\right. \\
& \text { 1) } \sqrt{\left(q^{2}-4 p r\right) /\left(m^{4}+14 m^{2}+1\right)}, a_{1}=0, a_{2}=-\left(3 m^{2} / p\right) \sqrt{\left(q^{2}-4 p r\right) /\left(m^{4}+14 m^{2}+1\right)}, \\
& b_{1}=0, b_{2}= \pm(3 m / p) \sqrt{\left(q^{2}-4 p r\right) /\left(m^{4}+14 m^{2}+1\right)}, \\
& v(\xi)=-\frac{q}{2 p}+\frac{1}{2 p}\left(m^{2}+1\right) \sqrt{\frac{q^{2}-4 p r}{m^{4}+14 m^{2}+1}} \\
& -\frac{3 m^{2}}{p} \sqrt{\frac{q^{2}-4 p r}{m^{4}+14 m^{2}+1}} \mathrm{sn}^{2}\left(\sqrt{-1} \sqrt[4]{\frac{q^{2}-4 p r}{m^{4}+14 m^{2}+1}} \xi \mid m\right) \\
& \pm \frac{3 m}{p} \sqrt{\frac{q^{2}-4 p r}{m^{4}+14 m^{2}+1}} \mathrm{cn}\left(\sqrt{-1} \sqrt[4]{\frac{q^{2}-4 p r}{m^{4}+14 m^{2}+1}} \xi \mid m\right) \\
& \times \operatorname{dn}\left(\sqrt{-1} \sqrt[4]{\frac{q^{2}-4 p r}{m^{4}+14 m^{2}+1}} \xi \mid m\right)
\end{aligned}
$$




$$
\begin{gathered}
\text { (ii) } k=\sqrt[4]{\left(q^{2}-4 p r\right) /\left(m^{4}+14 m^{2}+1\right)}, \quad a_{0}=-q / 2 p-(1 / 2 p)\left(m^{2}+\right. \\
\text { 1) } \sqrt{\left(q^{2}-4 p r\right) /\left(m^{4}+14 m^{2}+1\right)}, a_{1}=0, a_{2}=\left(3 m^{2} / p\right) \sqrt{\left(q^{2}-4 p r\right) /\left(m^{4}+14 m^{2}+1\right)} \\
b_{1}=0, b_{2}= \pm\left(3 m^{2} / p\right) \sqrt{\left(q^{2}-4 p r\right) /\left(m^{4}+14 m^{2}+1\right)} \\
v(\xi)=-\frac{q}{2 p}-\frac{1}{2 p}\left(m^{2}+1\right) \sqrt{\frac{q^{2}-4 p r}{m^{4}+14 m^{2}+1}} \\
-\frac{3 m^{2}}{p} \sqrt{\frac{q^{2}-4 p r}{m^{4}+14 m^{2}+1}} \mathrm{sn}^{2}\left(\sqrt[4]{\frac{q^{2}-4 p r}{m^{4}+14 m^{2}+1}} \xi \mid m\right) \\
\pm \frac{3 m}{p} \sqrt{\frac{q^{2}-4 p r}{m^{4}+14 m^{2}+1}} \mathrm{cn}\left(\sqrt[4]{\frac{q^{2}-4 p r}{m^{4}+14 m^{2}+1}} \xi \mid m\right) \operatorname{dn}\left(\sqrt[4]{\frac{q^{2}-4 p r}{m^{4}+14 m^{2}+1}} \xi \mid m\right) .
\end{gathered}
$$

We may obtain other solutions by making use of the dn-nd method.

\subsection{KdV Equation}

This is the equation

$$
u_{t}+6 u u_{x}+u_{x x x}=0
$$

If $u=v(\xi), \xi=x+\lambda t+\xi_{0}$, this equation takes the form

$$
\lambda v^{\prime}(\xi)+6 v(\xi) v^{\prime}(\xi)+v^{\prime \prime \prime}(\xi)=0 .
$$

Integrating this equation w.r.t. $\xi$ yields

$$
v^{\prime \prime}(\xi)=-3 v^{2}(\xi)+\lambda v(\xi)+C,
$$

where $C$ is the constant of integration. Equation (4.30) has the form (4.15) with

$$
p=-3, \quad q=\lambda, \quad r=C .
$$

Exact solutions to KdV equation may be derived from (4.18)-(4.25) and (4.31).

The KdV equation may also be solved by the Weierstrass elliptic functions method.

\subsection{Gardner Equation}

This equation reads

$$
u_{t}+\alpha u u_{x}+\beta u^{2} u_{x}+\gamma u_{x x x}=0, \quad \beta \gamma \neq 0
$$


This equation reduces to $\mathrm{mKdV}$ equation if we apply the similarity transformations

$$
\tilde{t}=\gamma t, \quad \tilde{x}=x+\frac{\alpha^{2}}{4 \beta} t, \quad \tilde{u}=\sqrt{\frac{|\beta|}{|\gamma|}}\left(u+\frac{\alpha}{2 \beta}\right)
$$

to the Gardner equation (4.32). We obtain the $\mathrm{mKdV}$ equation

$$
\tilde{u}_{\tilde{t}}+\varepsilon \tilde{u}_{\tilde{x}}^{2} \tilde{u}+\gamma \tilde{u}_{\tilde{x} \tilde{x} \tilde{x}}=0
$$

where $\varepsilon=\operatorname{sign}(\beta \gamma)= \pm 1$.

This means that if $\tilde{u}=\tilde{u}(\tilde{t}, \tilde{x})$ is a solution to the mKdV equation, then the function defined by

$$
u=u(t, x)=\sqrt{\frac{|\gamma|}{|\beta|}} \tilde{u}\left(\gamma t, x+\frac{\alpha^{2}}{4 \beta} t\right)-\frac{\alpha}{2 \beta}
$$

is a solution to Gardner equation (4.32). For solutions to MkdV equation, see [6].

\subsection{Boussinesq Equation}

This equation reads

$$
u_{t t}+\alpha u u_{x x}+\alpha u_{x}^{2}+\beta u_{x x x x}=0, \quad \alpha \beta \neq 0
$$

After the traveling wave transformation $u=v(\xi), \xi=x+\lambda t+\xi_{0}$ and integrating twice w.r.t $\xi$, we obtain the following ODE:

$$
v^{\prime \prime}(\xi)=-\frac{\alpha}{2 \beta} v^{2}(\xi)-\frac{\lambda^{2}}{\beta} v(\xi)-\frac{D \xi+C}{\beta}
$$

where $C$ and $D$ are the constants of integration. Setting $D=0$, we obtain an equation of the form (4.15) with

$$
p=-\frac{\alpha}{2 \beta}, \quad q=-\frac{\lambda^{2}}{\beta}, \quad r=-\frac{C}{\beta} .
$$

As we can see, exact solutions to the Boussinesq equation are calculated from (4.18)-(4.25) and (4.38). 


\subsection{Symmetric Regular Long Wave Equation}

This equation is defined by

$$
u_{t t}+u_{x x}+u u_{x t}+u_{x} u_{t}+u_{x x t t}=0
$$

Let $u=v(\xi), \xi=x+\lambda t+\xi_{0}$. Applying this transformation and integrating twice w.r.t. $\xi$, we obtain the ODE

$$
v^{\prime \prime}(\xi)=-\frac{1}{2 \lambda} v^{2}(\xi)-\frac{\lambda^{2}+1}{\lambda^{2}} v(\xi)-\frac{D \xi+C}{\lambda^{2}},
$$

where $C$ and $D$ are the constants of integration. Setting $D=0$, we obtain an equation of the form (4.15) with

$$
p=-\frac{1}{2 \lambda}, \quad q=-\frac{\lambda^{2}+1}{\lambda^{2}}, \quad r=-\frac{C}{\lambda^{2}} .
$$

Thus, exact solutions to symmetric regular long wave equation are easily found from (4.18)(4.25) taking into account (4.41).

\subsection{Generalized Shallow Water Wave Equation}

This equation is given by

$$
u_{x x t t}+\alpha u_{x} u_{x t}+\beta u_{t} u_{x x}-u_{x t}-u_{x x}=0 .
$$

Let

$$
u=u(x, t)=V(\xi), \quad V(\xi)=\int v(\xi) d \zeta, \xi=x+\lambda t .
$$

Substituting (4.43) into (4.42) and integrating once w.r.t. $\xi$, we obtain

$$
v^{\prime \prime}(\xi)=-\frac{\alpha+\beta}{2 \lambda} v^{2}(\xi)+\frac{\lambda+1}{\lambda^{2}} v(\xi)+\frac{C}{\lambda^{2}},
$$

where $C$ is the constant of integration. Equation (4.15) has the form (4.15) with

$$
p=-\frac{\alpha+\beta}{2 \lambda}, \quad q=\frac{\lambda+1}{\lambda^{2}}, \quad r=\frac{C}{\lambda^{2}} .
$$

It is evident that we may find exact solutions to generalized shallow water wave equation (4.42) from (4.18)-(4.25) for the choices given by (4.45). 


\subsection{Klein-Gordon Equation with Quadratic Nonlinearity}

The general Klein-Gordon equation has the form

$$
u_{t t}-\alpha^{2} u_{x x}+\beta u+f(u)=0 .
$$

In the case when $f(u)=-\gamma u^{2}$ we obtain the so-called Klein-Gordon equation with quadratic nonlinearity:

$$
u_{t t}-\alpha^{2} u_{x x}+\beta u-\gamma u^{2}=0
$$

Let $u=v(\xi), \xi=x+\lambda t+\xi_{0}$. After this traveling wave transformation, (4.47) reduces to

$$
v^{\prime \prime}(\xi)=-\frac{\gamma}{\alpha^{2}-\lambda^{2}} v^{2}(\xi)+\frac{\beta}{\alpha^{2}-\lambda^{2}} v(\xi), \quad \alpha^{2} \neq \lambda^{2},
$$

which is an equation of the form (4.15) with

$$
p=-\frac{\gamma}{\alpha^{2}-\lambda^{2}}, \quad q=\frac{\beta}{\alpha^{2}-\lambda^{2}}, \quad r=0
$$

Again, exact solutions to (4.47) are obtained from (4.18)-(4.25) taking into account (4.49).

\subsection{Fitzhugh-Nagumo-Huxley Equation}

This equation reads

$$
u_{t}-u_{x x}+u(1-u)(\alpha-u)=0, \quad \alpha=\text { const. }
$$

This equation is an important model in the study of neuron axon [7]. Let

$$
u=u(x, t)=v(\xi), \quad \xi=x+\lambda t+\xi_{0} .
$$

The corresponding ODE is

$$
v^{\prime \prime}(\xi)-\lambda v^{\prime}(\xi)-v(\xi)(v(\xi)-1)(v(\xi)-\alpha)=0 .
$$

Application of the sn-ns method gives only trivial solutions since we get $\lambda=0$. Instead, we may use other methods. If we apply the tanh-coth method or the exp method, we obtain nontrivial solutions. Indeed, balancing gives $n=1$. Following the tanh-coth method, we try the ansatz

$$
v(\xi)=a_{0}+a_{1} \tanh (k \xi)+b_{1} \operatorname{coth}(k \xi) .
$$


Inserting (4.53) into (4.50) and solving the corresponding algebraic system gives the following solutions to (4.50):

$$
\begin{gathered}
u(x, t)=\frac{1}{2}\left(1+\tanh \left[\frac{1}{2 \sqrt{2}}\left(x-\frac{2 \alpha-1}{\sqrt{2}} t+\xi_{0}\right)\right]\right), \\
u(x, t)=\frac{1}{2}\left(1+\operatorname{coth}\left[\frac{1}{2 \sqrt{2}}\left(x-\frac{2 \alpha-1}{\sqrt{2}} t+\xi_{0}\right)\right]\right), \\
u(x, t)=\frac{1}{4}\left(2+\tanh \left[\frac{1}{4 \sqrt{2}}\left(x-\frac{2 \alpha-1}{\sqrt{2}} t+\xi_{0}\right)\right]+\operatorname{coth}\left[\frac{1}{4 \sqrt{2}}\left(x-\frac{2 \alpha-1}{\sqrt{2}} t+\xi_{0}\right)\right]\right), \\
u(x, t)=\frac{\alpha}{2}\left(1+\tanh \left[\frac{\alpha}{2 \sqrt{2}}\left(x-\frac{2-\alpha}{\sqrt{2}} t+\xi_{0}\right)\right]\right), \\
u(x, t)=\frac{\alpha}{2}\left(1+\operatorname{coth}\left[\frac{\alpha}{2 \sqrt{2}}\left(x-\frac{2-\alpha}{\sqrt{2}} t+\xi_{0}\right)\right]\right), \\
u(x, t)=\frac{\alpha}{4}\left(2+\tanh \left[\frac{\alpha}{4 \sqrt{2}}\left(x-\frac{2 \alpha-1}{\sqrt{2}} t+\xi_{0}\right)\right]+\operatorname{coth}\left[\frac{\alpha}{4 \sqrt{2}}\left(x-\frac{2 \alpha-1}{\sqrt{2}} t+\xi_{0}\right)\right]\right) .
\end{gathered}
$$

\subsection{Double Sine-Gordon Equation}

Our last example deals with the double sine-Gordon equation. This equation in a normalized form reads

$$
u_{t t}-u_{x x}+\sin (u)-\frac{1}{2} \sin (2 u)=0 .
$$

This equation is an important model in the study of the DNA molecule [8].

The application of the tanh-coth method gives only the trivial solution $u=0$. If we apply the sn-ns method, we obtain nontrivial solutions. Indeed, let

$$
u=2 \arctan (v), \quad v=v(\xi), \quad \xi=x+\lambda t+\xi_{0} .
$$

Inserting ansatz (4.56) into (4.55) gives the ODE

$$
2 v^{3}(\xi)-2\left(\lambda^{2}-1\right) v(\xi)\left(v^{\prime}(\xi)\right)^{2}+\left(\lambda^{2}-1\right) v^{\prime \prime}(\xi)+\left(\lambda^{2}-1\right) v^{2}(\xi) v^{\prime \prime}(\xi)=0
$$

Let

$$
v(\xi)=a_{0}+a_{1} \operatorname{sn}(k \xi \mid m)+b_{1} \mathrm{~ns}(k \xi \mid m) .
$$

Substituting (4.58) into (4.57) and solving the corresponding algebraic system gives the following solutions to (4.55): 
(i) $a_{0}=0, a_{1}=(\sqrt{2} / 2) \sqrt{m^{2}+1} \sqrt{-1}, b_{1}=0, \lambda=\sqrt{k^{2}\left(m^{2}-1\right)^{2}-2\left(m^{2}+1\right)} / k\left(m^{2}-1\right)$,
$v(\xi)=\frac{\sqrt{2}}{2} \sqrt{m^{2}+1} \sqrt{-1} \operatorname{sn}\left(k\left(x+\frac{\sqrt{k^{2}\left(m^{2}-1\right)^{2}-2\left(m^{2}+1\right)}}{k\left(m^{2}-1\right)} t+\xi_{0}\right) \mid m\right)$,

(ii) $a_{0}=0, a_{1}=0, b_{1}=(\sqrt{2} / 2 m) \sqrt{m^{2}+1} \sqrt{-1}, \lambda=\sqrt{k^{2}\left(m^{2}-1\right)^{2}-2\left(m^{2}+1\right)} / k\left(m^{2}-1\right)$, $v(\xi)=\frac{\sqrt{2}}{2 m} \sqrt{m^{2}+1} \sqrt{-1} \mathrm{~ns}\left(k\left(x+\frac{\sqrt{k^{2}\left(m^{2}-1\right)^{2}-2\left(m^{2}+1\right)}}{k\left(m^{2}-1\right)} t+\xi_{0}\right) \mid m\right)$

(iii) $a_{0}=0, a_{1}=\sqrt{m\left(m^{2}-6 m+1\right)} / 2 \sqrt{2}(m-1), b_{1}=-\sqrt{m\left(m^{2}-6 m+1\right)} / 2 \sqrt{2} m(m-$ $1), \lambda=-\sqrt{k^{2}(m+1)^{4}-2\left(m^{2}-6 m+1\right)} / k(m+1)^{2}$,

$$
\begin{gathered}
v(\xi)=\frac{\sqrt{m\left(m^{2}-6 m+1\right)}}{2 \sqrt{2}(m-1)} \operatorname{sn}(k \xi \mid m)-\frac{\sqrt{m\left(m^{2}-6 m+1\right)}}{2 \sqrt{2 m(m-1)}} \mathrm{ns}(k \xi \mid m), \\
\xi=x-\frac{\sqrt{k^{2}(m+1)^{4}-2\left(m^{2}-6 m+1\right)}}{k(m+1)^{2}} t+\xi_{0} .
\end{gathered}
$$

\section{Comparison with Other Methods to Solve Nonlinear PDEs}

There are some other powerful and systematical approaches for solving nonlinear partial differential equations, such as the expansion along the integrable ODE [9, 10], the transformed rational function method [11], and the multiple expfunction method [12]. Even about linear DEs, there is some recent study on solution representations [13] and the linear superposition principle has been applied to bilinear equations [14].

\subsection{Multiple Exp Function Method}

Let us consider the following equation:

$$
u_{t}+u_{x x x x x}+30 u u_{x x x}+30 u_{x} u_{x x}+180 u^{2} u_{x}=0
$$

Equation (5.1) is also called the Sawada-Kotera equation [15]. In a recent work [16], the authors obtained multisoliton solutions to (5.1) by using Hirota's bilinear approach.

Introducing the potential $w$, defined by

$$
u=w_{x},
$$


(5.1) may be written in the form

$$
w_{t x}+w_{x x x x x x}+30 w_{x} w_{x x x x}+30 w_{x x} w_{x x x}+180 w_{x}^{2} w_{x x}=0
$$

Integrating (5.3) once w.r.t $x$ and taking the constant of integration equal to zero, the following partial differential equation is obtained:

$$
w_{t}+60 w_{x}^{3}+30 w_{x} w_{x x x}+w_{x x x x x}=0 .
$$

We will call (5.4) the potential Caudrey-Dodd-Gibbon equation associated with (5.1).

In view of the multiple exp method one-soliton solutions to (5.4) have the form

$$
w(x, t)=\frac{a_{0}+a_{1} \exp (\eta)}{1+b_{1} \exp (\eta)}, \quad \eta=\eta(x, t)=k x-\omega t
$$

where $a_{0}, a_{1}$, and $b_{1}$ are some constants to be determined. Inserting ansatz (5.5) into (5.4) and simplifying, we obtain the following polynomial equation in the variable $\zeta=\exp (\eta)$ :

$$
\begin{aligned}
k^{5}- & \omega+2\left(-15 a_{0} b_{1} k^{4}+15 a_{1} k^{4}-13 b_{1} k^{5}-2 b_{1} \omega\right) \zeta \\
& +6\left(20 a_{0} b_{1}^{2} k^{4}-20 a_{1} b_{1} k^{4}+10 a_{0}^{2} b_{1}^{2} k^{3}-20 a_{0} a_{1} b_{1} k^{3}+10 a_{1}^{2} k^{3}+11 b_{1}^{2} k^{5}-b_{1}^{2} \omega\right) \zeta^{2} \\
& +2 b_{1}^{2}\left(-15 a_{0} b_{1} k^{4}+15 a_{1} k^{4}-13 b_{1} k^{5}-2 b_{1} \omega\right) \zeta^{3}+b_{1}^{4}\left(k^{5}-\omega\right) \zeta^{4}=0 .
\end{aligned}
$$

Equating the coefficients of different powers of $\zeta$ to zero gives an algebraic system. Solving it with either Mathematica 8 or Maple 15, we obtain

$$
w=k^{5}, \quad a_{1}=b_{1}\left(a_{0}+k\right) .
$$

Thus, the following is a one-soliton solution (or one wave solution in Ma's terminology) to (5.4):

$$
w(x, t)=\frac{a_{0}+b_{1}\left(a_{0}+k\right) \exp (\eta)}{1+b_{1} \exp (\eta)}, \quad \eta=k x-k^{5} t
$$

In view of (5.2) one-soliton solution to Caudrey-Dodd-Gibbon equation (5.1) is

$$
u(x, t)=\frac{b_{1} k^{2} \exp \left(k x-k^{5} t\right)}{\left(1+b_{1} \exp \left(k x-k^{5} t\right)\right)^{2}} .
$$

Observe that solution (5.9) is the same solution obtained in [16] by using Hirota's bilinear method. We conclude that Hirota's method and multiple exp method give the same result 
for one-soliton solutions. Let us examine two-soliton solutions. In view of Ma's method the two-soliton solutions to (5.4) are of the form

$$
\begin{gathered}
w(x, t)=\frac{k_{1} \eta_{1}+k_{2} \eta_{2}+R\left(k_{1}+k_{2}\right) \eta_{1} \eta_{2}}{\Delta+\eta_{1}+\eta_{2}+R \eta_{1} \eta_{2}}, \quad \Delta \in\{0,1\}, \\
\eta_{1}=\exp \left(k_{1} x-\omega_{1} t\right), \quad \eta_{2}=\exp \left(k_{2} x-\omega_{2} t\right) .
\end{gathered}
$$

Let $\Delta=1$. Inserting ansatz (5.10) into (5.4) and simplifying, we obtain the following polynomial equation w.r.t the variables $\zeta_{1}=\exp \left(\eta_{1}\right)$ and $\zeta_{2}=\exp \left(\eta_{2}\right)$ :

$$
\begin{aligned}
c_{1}^{2} c_{2} & k_{2} R\left(k_{2}^{5}-\omega_{2}\right) \zeta_{1}^{2} \zeta_{2}+c_{1} c_{2}^{2} k_{1} R\left(k_{1}^{5}-\omega_{1}\right) \zeta_{1} \zeta_{2}^{2} \\
& +\left(c_{1} c_{2}\left(k_{1}+k_{2}\right)\left(k_{1}^{5}+5 k_{2} k_{1}^{4}+10 k_{2}^{2} k_{1}^{3}+10 k_{2}^{3} k_{1}^{2}+5 k_{2}^{4} k_{1}+k_{2}^{5}-\omega_{1}-\omega_{2}\right) R\right. \\
& \left.+c_{1} c_{2}\left(k_{1}-k_{2}\right)\left(k_{1}^{5}-5 k_{2} k_{1}^{4}+10 k_{2}^{2} k_{1}^{3}-10 k_{2}^{3} k_{1}^{2}+5 k_{2}^{4} k_{1}-k_{2}^{5}-\omega_{1}+\omega_{2}\right)\right) \zeta_{1} \zeta_{2} \\
& +c_{1} k_{1}\left(k_{1}^{5}-\omega_{1}\right) \zeta_{1}+c_{2} k_{2}\left(k_{2}^{5}-\omega_{2}\right) \zeta_{2}=0 .
\end{aligned}
$$

Equating the coefficients of $\zeta_{1}^{2} \zeta_{2}, \zeta_{1} \zeta_{2}^{2}, \zeta_{1} \zeta_{2}, \zeta_{1}$, and $\zeta_{2}$ to zero gives a system of algebraic equations. Solving it, we obtain the following nontrivial solution:

$$
w_{1}=k_{1}^{5}, \quad w_{2}=k_{2}^{5}, \quad R=\frac{\left(k_{1}-k_{2}\right)^{2}\left(k_{1}^{2}-k_{1} k_{2}+k_{2}^{2}\right)}{\left(k_{1}+k_{2}\right)^{2}\left(k_{1}^{2}+k_{1} k_{2}+k_{2}^{2}\right)}
$$

We see that solution (5.12) coincides with solutions obtained in [16]. The same is valid if we set $\Delta=0$. We conclude that Ma's method does not give any new solutions compared with Hirota's method.

\subsection{The Transformed Rational Function Method}

Given a nonlinear ODE in the unknown $u=u(x, t), u=u(x, y, t)$ or $u=u(x, y, z, t)$. we search for traveling wave solutions determined by

$$
\begin{gathered}
u^{(r)}(\xi)=v(\eta) \\
v(\eta)=\frac{p(\eta)}{q(\eta)}=\frac{p_{m} \eta^{m}+p_{m-1} \eta^{m-1}+\cdots+p_{0}}{q_{n} \eta^{n}+q_{n-1} \eta^{n-1}+\cdots+q_{0}}
\end{gathered}
$$

where $m$ and $n$ are two natural numbers and $p_{i}(0 \leq i \leq m), q_{j}(0 \leq j \leq n)$ are normally constants but could be functions of the independent variables and $\eta$ is a solution to equation

$$
A \eta^{\prime \prime}+B \eta^{\prime}+C \eta^{2}+D \eta+E=0
$$


with $A, B, C, D$, and $E$ being some constants. Observe that equations

$$
\begin{gathered}
\eta^{\prime}=\eta^{2}, \\
\eta^{\prime}=\alpha+\eta^{2} \quad \text { (Riccati equation), } \\
v^{\prime \prime}(\xi)=p v^{2}(\xi)+q v(\xi)+r \quad \text { (quadratic Duffing equation (4.15)) }
\end{gathered}
$$

are particular cases of (5.14). This method was applied in [11] to solve the $(3+1)$ - dimensional Jimbo-Miwa equation

$$
u_{x x x y}+3 u_{y} u_{x x}+3 u_{x} u_{x y}+2 u_{y t}-3 u_{x z}=0
$$

which converts into the nonlinear ODE

$$
a^{3} b u^{(4)}+6 a^{2} b u^{\prime} u^{\prime \prime}-(2 b w+3 a c) u^{\prime \prime}=0
$$

after the traveling wave transformation

$$
u(x, y, z, t)=u(\xi), \quad \xi=a x+b y+c z-\omega t
$$

If we integrate (5.14) once w.r.t $x$, then the following equation follows:

$$
v^{\prime \prime}(\xi)=-\frac{3}{a} v^{2}(\xi)+\frac{2 b \omega+3 a c}{a^{3} b} v(\xi), \quad \text { where } u(\xi)=\int v(\xi) d \xi .
$$

Observe that (5.20) is a quadratic Duffing equation (5.16) with $p=-3 / a, q=(2 b \omega+3 a c) / a^{3} b$, and $r=0$.

Solutions to this equation are given by (4.18)-(4.27). These solutions were not reported in [11]. On the other hand, it is clear from (4.24) that the sn-ns method covers the solutions obtained in [11].

\section{Conclusions}

We successfully obtained exact solutions for some important physical models by techniques based on projective equations. Mainly, we have used the sn-ns method. In our opinion, this is the most appropriate of all methods we have studied since it provides elliptic function solutions as well as trigonometric and hyperbolic solutions. However, in the cases when the sn-ns method does not work (this occurs for the Fitzhugh-Nagumo-Huxley equation), we may try other methods, such as the tanh-coth method. On the other hand, there are some equations for which the tanh-coth method gives only trivial solutions (this is the case of the double sine-Gordon equation).

We think that some of the results we obtained are new in the open literature. Other results concerning exact solutions of nonlinear PDEs may be found in [6, 15, 17-52]. 


\section{References}

[1] E. Fan and Y. C. Hon, "Generalized tanh method extended to special types of nonlinear equations," Zeitschrift fur Naturforschung, vol. 57, no. 8, pp. 692-700, 2002.

[2] A.-M. Wazwaz, "The extended tanh method for new solitons solutions for many forms of the fifthorder KdV equations," Applied Mathematics and Computation, vol. 184, no. 2, pp. 1002-1014, 2007.

[3] J.-H. He and L.-N. Zhang, "Generalized solitary solution and compacton-like solution of the JaulentMiodek equations using the Exp-function method," Physics Letters A, vol. 372, no. 7, pp. 1044-1047, 2008.

[4] R. Conte and M. Musette, "Link between solitary waves and projective Riccati equations," Journal of Physics A, vol. 25, no. 21, pp. 5609-5623, 1992.

[5] W.-X. Ma and M. Chen, "Direct search for exact solutions to the nonlinear Schrödinger equation," Applied Mathematics and Computation, vol. 215, no. 8, pp. 2835-2842, 2009.

[6] C. A. Gómez, A. H. Salas, and B. Acevedo Frias, "Exact solutions to KdV6 equation by using a new approach of the projective Riccati equation method," Mathematical Problems in Engineering, Article ID 797084, 10 pages, 2010.

[7] A. Scott, Neuroscience-A Mathematical Primer, Springer-Verlag, New York, NY, USA, 2002.

[8] L. V. Yakushevich, Nonlinear Physics of DNA, Wiley, NewYork, NY, USA, 1998.

[9] W. X. Ma, H. Wu, and J. He, "Partial differential equations possessing Frobenius integrable decompositions," Physics Letters A, vol. 364, no. 1, pp. 29-32, 2007.

[10] W. Ma, "Comment on the $3+1$ dimensional Kadomtsev-Petviashvili equations," Communications in Nonlinear Science and Numerical Simulation, vol. 16, no. 7, pp. 2663-2666, 2011.

[11] W. X. Ma and J. H. Lee, "A transformed rational function method and exact solutions to the $3+1$ dimensional Jimbo-Miwa equation," Chaos, Solitons and Fractals, vol. 42, no. 3, pp. 1356-1363, 2009.

[12] W. X. Ma, T. Huang, and Y. Zhang, "A multiple exp-function method for nonlinear differential equations and its application," Physica Scripta, vol. 82, no. 6, Article ID 065003, 2010.

[13] W. X. Ma, X. Gu, and L. Gao, "A note on exact solutions to linear differential equations by the matrix exponential," Advances in Applied Mathematics and Mechanics, vol. 1, no. 4, pp. 573-580, 2009.

[14] W. X. Ma and E. Fan, "Linear superposition principle applying to Hirota bilinear equations," Computers and Mathematics with Applications, vol. 61, no. 4, pp. 950-959, 2011.

[15] A. Salas, "Some solutions for a type of generalized Sawada-Kotera equation," Applied Mathematics and Computation, vol. 196, no. 2, pp. 812-817, 2008.

[16] A. H. Salas, O. G. Hurtado, and J. E. Castillo, “Computing multi-soliton solutions to Caudrey-DoddGibbon equation by hirota's method," International Journal of Physical Sciences, vol. 6, no. 34, pp. 7729 7737, 2011.

[17] A. H. Salas, C. A. Gómez S, and B. Acevedo Frias, "Computing exact solutions to a generalized LaxSawada-Kotera-Ito seventh-order KdV equation," Mathematical Problems in Engineering, Article ID 524567, 7 pages, 2010.

[18] A. H. Salas and C. A. Gómez S, "Exact solutions for a third-order KdV equation with variable coefficients and forcing term," Mathematical Problems in Engineering, Article ID 737928, 13 pages, 2009.

[19] A. H. Salas and C. A. Gómez S., "Application of the Cole-Hopf transformation for finding exact solutions to several forms of the seventh-order KdV equation," Mathematical Problems in Engineering, Article ID 194329, 14 pages, 2010.

[20] C. A. Gómez and A. H. Salas, "Exact solutions for the generalized BBM equation with variable coefficients," Mathematical Problems in Engineering, vol. 2010, Article ID 498249, 10 pages, 2010.

[21] A. Salas, C. A. Gómez, and J. Gonzalo Escobar Lugo, "Exact solutions for the general fifth order KdV equation by the extended tanh method," Journal of Mathematical Sciences, vol. 1, no. 2, pp. 305-310, 2008.

[22] C. A. Gómez and A. H. Salas, "Exact solutions for a new integrable system (KdV6)," Journal of Mathematical Sciences, vol. 1, no. 2, pp. 401-413, 2008.

[23] A. Salas and C. Gómez, "A practical approach to solve coupled systems of nonlinear PDE's," Journal of Mathematical Sciences, vol. 3, no. 1, pp. 101-107, 2009.

[24] C. A. Gomez and A. Salas, "Solutions for a class of fifth-order nonlinear partial differential system," Journal of Mathematical Sciences, vol. 3, no. 1, pp. 121-128, 2009. 
[25] A. H. Salas, "Symbolic computation of solutions for a forced Burgers equation," Applied Mathematics and Computation, vol. 216, no. 1, pp. 18-26, 2010.

[26] A. H. Salas, "Exact solutions for the general fifth KdV equation by the exp function method," Applied Mathematics and Computation, vol. 205, no. 1, pp. 291-297, 2008.

[27] C. A. Gómez and A. H. Salas, "The Cole-Hopf transformation and improved tanh-coth method applied to new integrable system (KdV6)," Applied Mathematics and Computation, vol. 204, no. 2, pp. 957-962, 2008.

[28] A. H. Salas, C. A. Gomez S., and J. E. Castillo Hernańdez, "New abundant solutions for the Burgers equation," Computers \& Mathematics with Applications, vol. 58, no. 3, pp. 514-520, 2009.

[29] C. A. Gómez and A. H. Salas, "The generalized tanh-coth method to special types of the fifth-order KdV equation," Applied Mathematics and Computation, vol. 203, no. 2, pp. 873-880, 2008.

[30] A. H. Salas, "New solutions to Korteweg-de Vries (KdV) equation by the Riccati equation expansion method," International Journal of Applied Mathematics, vol. 22, no. 7, pp. 1169-1177, 2009.

[31] C. A. Gómez, "Special forms of the fifth-order KdV equation with new periodic and soliton solutions," Applied Mathematics and Computation, vol. 189, no. 2, pp. 1066-1077, 2007.

[32] C. A. Gomez and A. H. Salas, "Exact solutions for a reaction-diffusion equation by using the generalized tanh method," Scientia et Technica, Universidad Tecnológica de Pereira, Risaralda-Colombia, vol. 13, no. 035, pp. 409-410, 2007.

[33] C. A. Gómez and A. H. Salas, "The variational iteration method combined with improved generalized tanh-coth method applied to Sawada-Kotera equation," Applied Mathematics and Computation, vol. 217, no. 4, pp. 1408-1414, 2010.

[34] C. A. Gómez and A. Salas, "Special symmetries to standard Riccati equations and applications," Applied Mathematics and Computation, vol. 216, no. 10, pp. 3089-3096, 2010.

[35] C. A. Gómez, A. H. Salas, and B. Acevedo Frias, "New periodic and soliton solutions for the Generalized BBM and Burgers-BBM equations," Applied Mathematics and Computation, vol. 217, no. 4, pp. 1430-1434, 2010.

[36] A. H. Salas, J. E. Castillo, and J. G. Escobar, "About the seventh-order Kaup-Kupershmidt equation and its solutions," Tech. Rep. 0809.2865, 2008.

[37] A. H. Salas, C. A. Gómez, and J. E. Castillo H., "New solutions for the modified generalized Degasperis-Procesi equation," Applied Mathematics and Computation, vol. 215, no. 7, pp. 2608-2615, 2009.

[38] A. H. Salas, "Some exact solutions for the Caudrey-Dodd-Gibbon equation," Tech. Rep. 0805.2969v2, 2008.

[39] A. H. Salas and C. A. Gómez, "El software Mathematica en la búsqueda de soluciones exactas de ecuaciones diferenciales no lineales en derivadas parciales mediante el uso de la ecuación de Riccati," in Memorias del Primer Seminario Internacional de Tecnologias en Educación Matemática, vol. 1, pp. 379387, Universidad Pedagógica Nacional, Santafé de Bogotá, Bogotá, Colombia, 2005.

[40] A. H. Salas, C. A. Gómez, and L. L. Palomá, "Travelling wave solutions to the reduced Ostrovsky equation," International Journal of Applied Mathematics, vol. 23, pp. 249-256, 2010.

[41] A. H. Salas, "Symbolic Computation of exact solutions to KdV equation," Canadian Applied Mathematics Quarterly, vol. 16, no. 4, 2008.

[42] A. H. Salas, C. A. Gómez, and J. E. Castillo, "Symbolic computation of solutions for the general fifthorder KdV equation," International Journal of Nonlinear Science, vol. 9, no. 4, pp. 394-401, 2010.

[43] C. A. Gómez and A. H. Salas, "A generalized Riccati method to solve NLPDEs," Far East Journal of Applied Mathematics, vol. 40, no. 2, pp. 89-175, 2010.

[44] J. E. Castillo, A. H. Salas, and J. G. Escobar, "Exact solutions for a nonlinear model," Applied Mathematics and Computation, vol. 217, pp. 1646-1651, 2010.

[45] A. H. Salas, "Exact solutions of coupled sineGordon equations," Nonlinear Analysis: Real World Applications, vol. 11, no. 5, pp. 3930-3935, 2010.

[46] A. H. Salas, "Computing solutions to a forced KdV equation," Nonlinear Analysis: Real World Applications, vol. 12, no. 2, pp. 1314-1320, 2011.

[47] A. H. Salas and C. A. Gómez, "Computing exact solutions for some fifth KdV equations with forcing term," Applied Mathematics and Computation, vol. 204, no. 1, pp. 257-260, 2008.

[48] A. H. Salas, "Exact solutions to mKdV equation with variable coefficients," Applied Mathematics and Computation, vol. 216, no. 10, pp. 2792-2798, 2010.

[49] A. H. Salas, "Computing exact solutions to a generalized Lax seventh-order forced KdV equation (KdV7)," Applied Mathematics and Computation, vol. 216, no. 8, pp. 2333-2338, 2010. 
[50] A. H. Salas and J. E. H. Castillo, "New exact solutions to sinh-cosh-Gordon equation by using techniques based on projective Riccati equations," Computers and Mathematics with Applications, vol. 61, no. 2, pp. 470-481, 2011.

[51] A. H. Salas and C. A. Gómez, "A note on the G0/G method and the improved tanh-coth method," Far East Journal of Applied Mathematics, vol. 48, no. 1, pp. 1-73, 2010.

[52] A. H. Salas and C. A. Gomez, "Exact Solutions for the KdV6 and mKdV6 equations via tanh, coth and sech methods," Applications and Applied Mathematics, vol. 5, no. 10, pp. 1504-1510, 2010. 


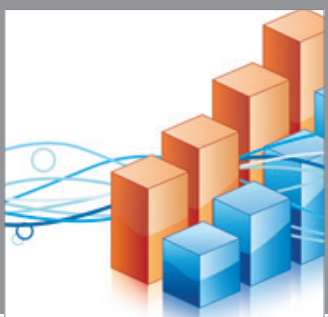

Advances in

Operations Research

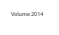

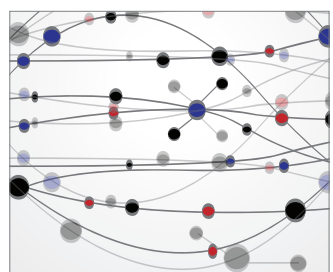

\section{The Scientific} World Journal
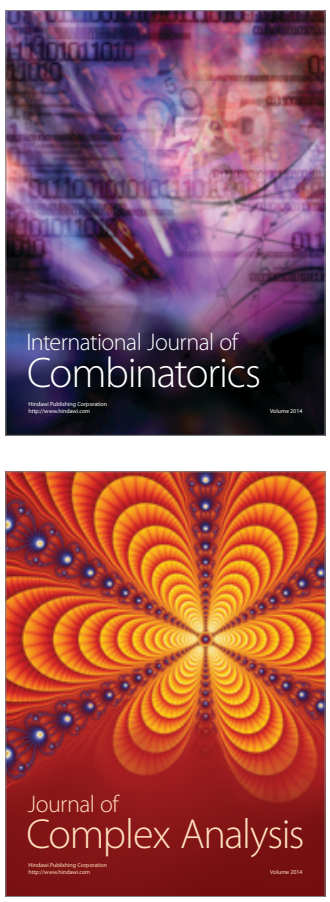

International Journal of

Mathematics and

Mathematical

Sciences
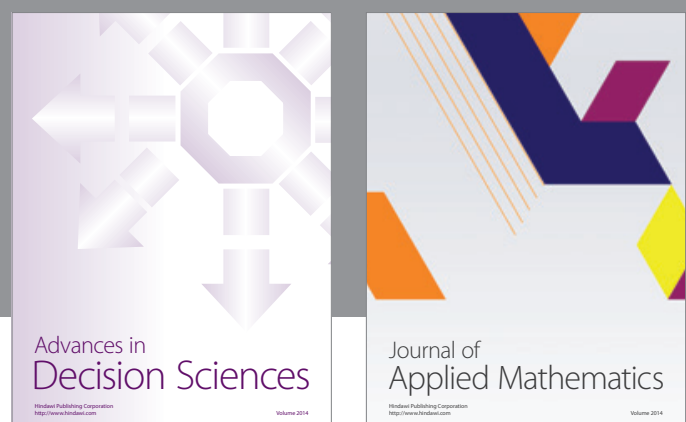

Journal of

Applied Mathematics
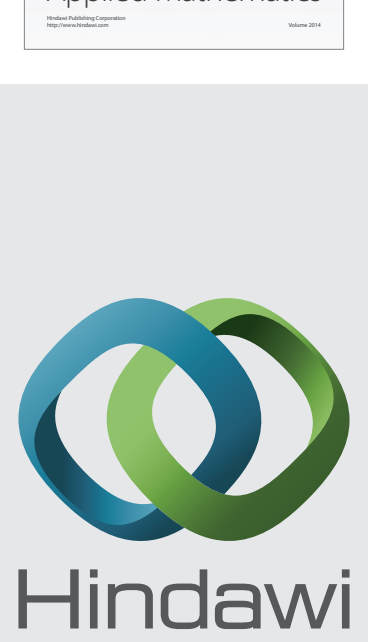

Submit your manuscripts at http://www.hindawi.com
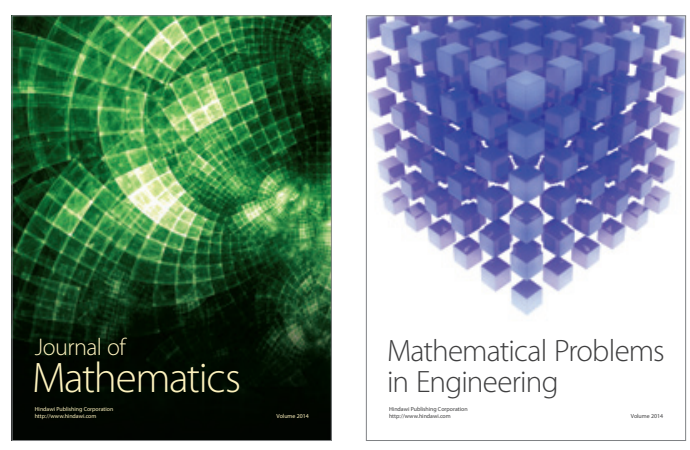

Mathematical Problems in Engineering
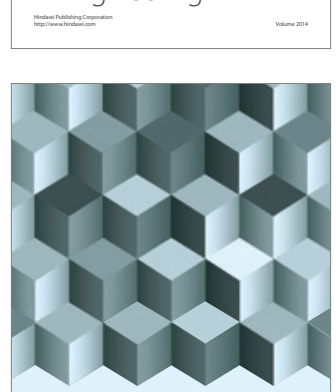

Journal of

Function Spaces
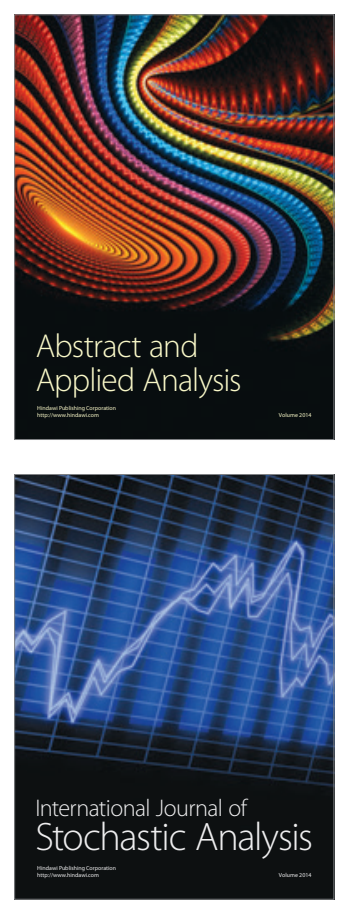

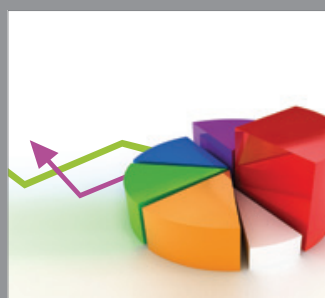

ournal of

Probability and Statistics

Promensencen
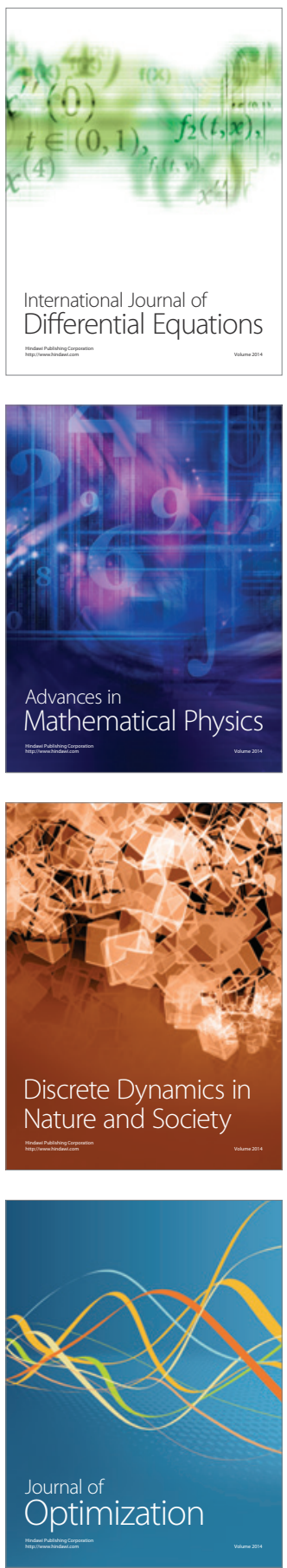